\title{
La séropositivité au féminin : du stigmate à la maladie chronique, une transition hypothétique
}

\author{
Marie-Ange SCHILTZ'
}

\begin{abstract}
Résumé ${ }^{1}$
L'introduction des traitements antirétroviraux contre le VIH/sida a induit une interrogation sur la possibilité que cette atteinte devienne une maladie comme une autre. Sur fond de cette aspiration à la banalisation, cet article se propose d'explorer l'acceptabilité de l'atteinte par le VIH. Le travail d'analyse s'appuie sur la comparaison des comportements et des trajectoires de vie des femmes ayant répondu à deux enquêtes, l'une auprès des personnes atteintes par le VIH, l'autre auprès d'un échantillon représentatif de la population féminine française discriminé en deux groupes, sur la base de l'état de santé des répondantes. En population générale, il apparaît que les parcours de vie affectifs et familiaux des femmes déclarant un handicap ou une atteinte grave de santé ne se distinguent guère de ceux des femmes bien portantes. Au contraire, la situation affective, matrimoniale et familiale des femmes atteintes par le $\mathrm{VIH}$ se caractérise par l'accélération des calendriers et la précarité des liens qu'elles établissent.
\end{abstract}

Mots clefs: femme, séropositivité, conjugalité, sexualité, stigmate, normalisation, maladie chronique.

L'introduction des traitements antirétroviraux, efficaces contre le $\mathrm{VIH} /$ sida a induit une interrogation sur la possibilité que cette atteinte devienne une maladie comme une autre $[1,2]$. Dans le contexte de cette aspiration à la banalisation d'une pathologie, entachée depuis le début d'un fort risque de discrimination, cet article explore l'acceptabilité de l'atteinte par le VIH. Dans quelle mesure la perception de cette caractéristique de santé interne à l'individu peut-elle s'améliorer, alors que s'estompe le caractère létal de l'atteinte? Dans quelle mesure d'autres caractéristiques sociales et privées attachées à des conduites et des modes de vie qui auraient exposé la femme à la contamination par le VIH sontelles susceptibles de les maintenir à l'écart de l'insertion? De nombreuses études sur l'exclusion ont montré l'importance des ruptures de trajectoires (chômage, deuil, divorce, migration,...) dans les risques de marginalisation et de solitude des personnes [3]. Dans le cadre de cette hypothèse de ruptures qui engendrent des mécanismes d'exclusion, nous nous proposons de caracté-

CAMS, CNRS, UMR 8169, CERMES, Villejuif. riser les parcours biographiques des femmes séropositives en termes d'insertions économique, affective et sexuelle. II s'agit de déterminer si la maladie, quelle qu'en soit la forme, est un facteur discriminant en soi ou dans quelle mesure les biographies et les modes de vie des femmes atteintes par le VIH se confondent ou se distinguent des autres pathologies graves.

Pour éclairer cette question, nous confronterons les modes de vie et les parcours affectifs et sexuels des femmes de nationalité française atteintes par le VIH à ceux de deux groupes de femmes issus d'un échantillon aléatoire représentatif de la population féminine de nationalité française qui ont été discriminés selon le critère d'une atteinte de santé grave et chronique.

\section{Méthodologie}

Ce travail d'analyse s'appuie sur les réponses des femmes à deux enquêtes représentatives, l'une menée en 2003 auprès des personnes atteintes par le VIH, l'enquête Anrs-Vespa sur les personnes infectées [4], l'autre réalisée en 2000 auprès d'un échantillon de la population féminine "Enquête nationale sur la violence envers les femmes en France " (Enveff) [5]. La comparaison porte sur les répondantes de nationalité française ${ }^{2}$, âgées de 20 à 59 ans, vivant en France métropolitaine, soit 534 femmes pour Vespa et 6291 pour l'Enveff. Tous les pourcentages présentés sont calculés sur la base des échantillons redressés.

Étant donné la gravité de l'infection à VIH, les comparaisons sont conduites sur la base de l'état de santé. Au sein de l'enquête Enveff, 1391 femmes ont répondu positivement à la question portant sur l'existence d'un "problème de santé chronique: maladie, invalidité ou handicap " et 1351 ont précisé la nature de l'atteinte. À partir de ces informations, il a été distingué un groupe de femmes qui déclarent un handicap ou une maladie grave et chronique (Enveff_2, $n=326$ ) d'un groupe référent composé de femmes qui ne déclarent rien ou simplement des « ennuis " ou

\footnotetext{
L'importance numérique et la spécificité des modes de vie des femmes de nationalité étrangère parmi l'échantillon de femmes séropositives nous a conduit à exclure cette population des analyses. En revanche, les françaises par acquisition dont le poids est équilibré dans les deux échantillons $(3,5 \%)$, sont retenues pour l'analyse.
} 
" accidents » de santé moins invalidants (Enveff_1, $n=5965$ ). Les cancers, la bronchite chronique, les rhumatismes inflammatoires, la maladie de Crohn... ont été classés en atteintes " graves ", La pertinence de ce recodage a été testée au regard de sa cohérence avec les réponses à d'autres questions sur le suivi médical des personnes dans les douze derniers mois.

Enfin, en raison de l'importance et de la "normalité » du processus de dégradation de l'état de santé lié au vieillissement, les comparaisons menées sont toutes modulées par l'âge dont il faut déterminer les classes. Un âge moyen des deux échantillons quasi similaire ( 40 ans pour Vespa versus 39 ans pour l'Enveff) cache en fait des disparités importantes. Parmi les femmes séropositives, la distribution des âges est plus concentrée autour de la moyenne: plus de la moitié d'entre elles ont entre 35 et 44 ans contre un peu plus du quart en population générale. Et dans l'Enveff, la moyenne d'âge est plus élevée parmi celles qui déclarent des problèmes graves de santé ( 44 ans contre 39 ans) : plus de la moitié d'entre elles ont plus de 45 ans. Pour la suite de l'analyse, nous proposons de mener les comparaisons selon les trois classes d'âge figurant dans le tableau 1, qui respectent les caractéristiques d'âge des trois groupes de femmes tout en conservant des effectifs suffisants pour l'analyse statistique.

Après une brève présentation de la situation économique, la comparaison se centrera sur les modes de vie affectifs et sexuels, les accidents de la vie (séparations, avortements, tentatives de suicide) et consommations " excessives " de produits psychoactifs.

Tableau 1

Répartition selon l'âge des femmes des trois échantillons

\begin{tabular}{lccc}
\hline \multicolumn{1}{c}{$\begin{array}{c}\text { Groupes d'âges } \\
\text { pour l'analyse }\end{array}$} & $\begin{array}{c}\text { Enveff_1 } \\
\mathbf{n = 5 9 6 5}\end{array}$ & $\begin{array}{c}\text { Enveff_2 } \\
\mathbf{n = 3 2 6}\end{array}$ & $\begin{array}{c}\text { Vespa } \\
\mathbf{n = 5 3 4}\end{array}$ \\
\hline 20-34 ans (les plus jeunes) & $38,6 \%$ & $18,3 \%$ & 21,1 \\
35-44 ans (d'âge moyen) & $26,9 \%$ & $29,1 \%$ & 53,8 \\
45-59 ans (les plus âgées) & $34,5 \%$ & $52,6 \%$ & 25,1 \\
\hline
\end{tabular}

Champ : ensemble des femmes de nationalité française âgées de 20 à 59 ans.

\section{Une situation économique problématique}

Des travaux antérieurs ont mis en évidence la situation particulièrement fragile des séropositifs face à l'emploi [6]. L'indicateur des niveaux comparés d'activité, de chômage et d'inactivité professionnelle donne une idée de la protection sociale ou, au contraire, de la précarité dans lesquelles s'inscrivent les trois populations de femmes.

Très tôt, par rapport à l'activité professionnelle, la situation des femmes atteintes par le VIH et, dans une moindre mesure, celle des femmes malades de l'Enveff, se caractérise par un taux de chômage élevé : respectivement 31 \% pour les femmes atteintes par le VIH et $20 \%$ pour celles avec atteinte grave de l'Enveff
Figure 1

\section{Proportion de femmes bénéficiant de minimas sociaux}

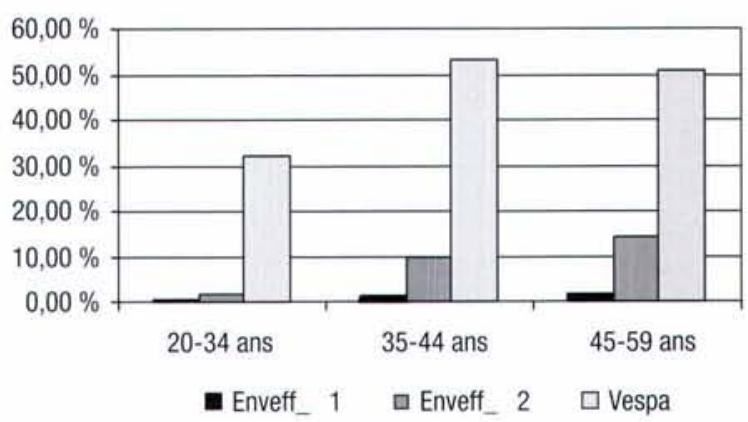

Champ : ensemble des femmes de nationalité française âgées de 20 à 59 ans.

contre $9 \%$ pour celles sans atteinte ${ }^{3}$. Par la suite, à partir de 45 ans, les femmes déclarant des pathologies graves se caractérisent par des taux d'inactivité élevés : $49 \%$ et $43 \%$ d'entre elles sont inactives contre $26 \%$ pour les femmes sans atteinte grave. II apparaît que couplée avec l'âge, l'atteinte grave, quelle qu'en soit la nature, induit une difficulté d'insertion professionnelle. Mais, si l'on s'en tient à la façon dont cette difficulté est prise en compte par l'administration alors la situation des femmes séropositives vis-à-vis de l'emploi prend une tournure très particulière. Certes, toutes les pathologies lourdes ouvrent des droits au revenu social (RMI, allocation d'adulte handicapé,...), mais l'ampleur de la prise en charge des femmes séropositives est sans commune mesure avec celle des femmes ayant d'autres problèmes de santé. En leur allouant relativement largement des minima sociaux à tout âge (figure 1), l'administration reconnaît leur situation de grande vulnérabilité sociale, ratifiant de fait, y compris pour les plus jeunes, leur difficulté, si ce n'est leur inaptitude à occuper un emploi.

En effet, ce " mince filet de sécurité », comme le nomme R Castel [7], destiné à compenser les difficultés durables de catégories de populations qui ne peuvent conduire avec un minimum d'indépendance leur existence en raison de la faiblesse de leurs ressources, supports et droits, contient le risque d'entériner - temporairement ou définitivement - l'incapacité des bénéficiaires à s'inscrire dans l'univers du travail et des protections qui lui sont attachées.

La précarité de la situation économique trouve écho dans la plus forte proportion, quel que soit leur âge, de femmes séropositives seules avec charge d'enfant : $19 \%$ contre respectivement $12 \%$ et $8 \%$. En effet, selon les statistiques du ministère de l'Emploi, du Travail et de la Cohésion sociale, les familles monoparentales sont les plus nombreuses à disposer de revenus inférieurs au seuil de pauvreté : $42 \%$ avant et $14 \%$ après transferts sociaux [8].

3 Afin de ne pas alourdir le texte, dans la suite de cet article, les références aux groupes de femmes selon leur état de santé se feront toujours dans le même ordre. 


\section{Une vie affective et familiale qui se fait et se défait constamment}

\section{Un calendrier précipité}

Dès leur plus jeune âge, et ce quel que soit l'indicateur considéré, les femmes de l'enquête Vespa se caractérisent par une indépendance et une initiation affective et sexuelle tôt acquises. Si l'on considère le fait d'habiter seule ou à deux comme un indicateur plausible de départ du foyer parental, alors les femmes séropositives sont autonomes plus tôt : dans la tranche d'âge 20-34 ans, 54 \% d'entre elles sont seules ou à deux dans leur logement contre respectivement $39 \%$ parmi les femmes déclarant une atteinte grave et $32 \%$ parmi celles sans atteinte grave dans l'Enveff. Bien d'autres critères témoignent d'une vie accélérée. Plus rapidement que les autres, ces femmes s'engagent dans la sexualité et la conjugalité, y compris parmi les plus jeunes : $2 \%$ de ces femmes atteintes par le VIH sont sans expérience sexuelle (contre respectivement $5 \%$ et $11 \%$ ) et seulement $1 \%$ déclarent n'avoir jamais eu de relation affective de plus de six mois (contre respectivement $10 \%$ et $23 \%$ ). Mais, pour beaucoup, cet engagement aura été de courte durée puisque dès cet âge, $27 \%$ d'entre elles sont désormais seules alors qu'elles avaient auparavant établi une relation suffisamment forte pour être mentionnée (contre respectivement $15 \%$ et $10 \%$ ) et $11 \%$ sont divorcées (contre respectivement $2 \%$ et $2 \%$ ). Avant 35 ans, presque la moitié des femmes séropositives a déjà fait l'expérience de ruptures répétées, confirmant ainsi l'instabilité de leur relation : $45 \%$ déclarent trois relations ou plus qui ont compté au cours de leur courte vie amoureuse alors qu'en population générale, au même âge, ces taux sont respectivement de $17 \%$ et $14 \%$. L'accélération déjà constatée des calendriers se lit également dans le domaine de la reproduction : toujours dans la tranche d'âge 20-34 ans, ces jeunes femmes sont plus souvent mères ( $53 \%$ contre respectivement $48 \%$ et $41 \%$ ). Seul le taux de femmes séropositives jeunes déclarant une relation privilégiée au moment de l'enquête ne diffère pas significativement de celui constaté en population générale $(73 \%$ contre respectivement $74 \%$ et $67 \%$ ), à ceci près que ce lien se caractérise par sa volatilité potentielle (moindres cohabitation et légalisation de la liaison, taux de ruptures et divorce plus importants).

\section{Pas de processus d'accumulation}

À la maturité, les relations affectives des femmes séropositives continuent à être marquées par le faible taux des unions légalisées et l'importance des relations non cohabitantes. Dans la tranche d'âge 35-44 ans, en population générale, I'union légalisée est la norme, au moins au sens statistique du terme (respectivement $64 \%$ et $69 \%$ parmi les femmes avec ou sans atteinte grave de l'Enveff sont mariées ou pacsées contre seulement $28 \%$ parmi celles de Vespa). Aussi n'est-il pas étonnant de constater un taux plus élevé de célibat parmi ces dernières : autour de la quarantaine, $48 \%$ d'entre elles ne se sont jamais mariées contre respectivement $19 \%$ et $20 \%$ dans l'enquête Enveff. Cependant, en raison de l'importance des relations non officialisées dans ce groupe, il faut bien se garder d'assimiler " célibat " et vie de "vieille fille ». En effet, l'absence de reconnaissance du lien n'est pas seulement d'ordre juridique : significativement plus souvent que les autres, les relations des femmes séropositives ne s'inscrivent pas toujours dans un quotidien partagé : entre 35 et 44 ans, $19 \%$ des femmes atteintes par le VIH sont dans une relation non cohabitante contre respectivement $8 \%$ et $4 \%$ en population générale. Par ailleurs, ces femmes se caractérisent par des liens moins pérennes : dans la tranche d'âge 35-44 ans, $24 \%$ des femmes séropositives sont séparées, divorcées ou veuves contre respectivement $18 \%$ et $10 \%$ en population générale ; $63 \%$ déclarent au moins « trois relations de plus de six mois " au cours de leur vie, alors qu'en population générale cette proportion est trois fois plus faible avec respectivement $21 \%$ et $17 \%$. Ces nombreuses ruptures induisent des taux de vie en solo plus élevés. Dans la tranche d'âge médiane, $32 \%$ des femmes séropositives vivent seules à la suite d'un divorce, une séparation ou un deuil contre respectivement $15 \%$ et $10 \%$ en population générale. Enfin, l'examen du nombre de personnes dans le foyer vient conforter l'idée que ces femmes ne sont pas dans une dynamique d'établissement familial. À l'âge où la vie en famille est de règle, on constate que les femmes séropositives vivent plus rarement dans un foyer comptant trois personnes ou plus ( $40 \%$ contre respectivement $71 \%$ et $85 \%$ ). Probablement en lien avec la précarité des situations matrimoniales, au-delà de 35 ans, la proportion des femmes atteintes par le VIH ayant plusieurs enfants n'augmente guère : dans la tranche $35-44$ ans, $23 \%$ déclarent deux enfants ou plus contre respectivement $70 \%$ et $73 \%$ en population générale.

Ce faisceau d'indicateurs met en évidence qu'à un âge où les femmes de l'échantillon en population générale sont dans une dynamique d'accumulation et de conservation des liens affectifs et familiaux, les trajectoires affectives et familiales de celles atteintes par le VIH sont très particulières, relevant plus, semble-t-il, d'une dynamique d'opportunités avec réitération des expériences. II apparaît qu'à la maturité, ces femmes ne sont pas dans un processus de consolidation et, de ce fait, ont des difficultés à se maintenir dans une unité familiale au sein d'une relation de couple monogame et stable qui représente toujours, dans nos sociétés, les horizons indépassables de la vie sexuelle et affective [9].

\section{Une solitude prématurée}

Avec le temps, effet d'âge et effet de générations se combinent : le nombre d'expériences affectives déclarées sur la vie, relativement stable ou en légère diminution malgré l'allongement de la " carrière " amoureuse, peut être interprété comme une raréfac- 
tion des nouvelles opportunités liée au vieillissement, éventuellement cumulée à un effet de génération auquel s'ajoute le facteur " oubli ". Dans chacune des populations, parmi les femmes les plus âgées, le nombre de relations affectives qui ont compté fléchit au regard de celui déclaré par les femmes d'âge moyen. Bien que la baisse soit générale, les écarts entre les femmes séropositives et les autres s'accentuent : dans la tranche d'âge 45-59 ans, 59 \% des femmes séropositives déclarent " trois relations ou plus ", contre respectivement $11 \%$ et $10 \%$ en population générale. De même, les écarts liés à l'isolement s'amplifient avec l'âge. Même si la solitude des femmes déclarant des atteintes graves de santé augmente plus rapidement que celle des femmes témoins, leur isolement est loin d'être aussi répandu que celui des femmes séropositives. Dans la tranche d'âge des 45-59 ans, la plus grande solitude de ces dernières ne fait plus de doute : $42 \%$ vivent seules dans un logement contre respectivement $15 \%$ et $6 \%$, et $44 \%$ d'entre elles n'ont pas de relation affective stable contre respectivement $28 \%$ et $18 \%$. Le taux de célibat après 50 ans, que les démographes qualifient de " célibat définitif », rare en population générale, concerne une forte minorité de femmes séropositives ( $18 \%$ contre respectivement $8 \%$ et $7 \%$ ). Enfin, comme condensé d'une vie qui se fait et se défait au gré de liens relativement éphémères, au-delà de 45 ans, $48 \%$ des femmes séropositives sont désormais " divorcées ", " séparées " ou " veuves " contre respectivement $28 \%$ et $19 \%$ en population générale.

En résumé, dans l'enquête Enveff, les parcours de vie affectifs et familiaux des femmes déclarant un handicap ou une atteinte grave de santé ne se distinguent guère de ceux des femmes bien portantes : malgré un calendrier légèrement accéléré, une moindre légalisation des liens affectifs et des incidents de parcours un peu plus fréquents, comme les femmes " ordinaires ", ces femmes s'engagent massivement dans la vie conjugale et familiale. À l'encontre de ces parcours cumulatifs, tous les indicateurs attestent la fragilité des liens amoureux des femmes atteintes par le VIH et laissent entrevoir une vie affective plus perturbée. Leur situation affective, matrimoniale et familiale se caractérise par l'accélération des calendriers et la précarité des liens qu'elles établissent : dans la tranche d'âge 20-34 ans, elles sont plus nombreuses que les autres à vivre seules dans un logement, à s'être déjà engagées dans une ou plusieurs relations électives, à avoir eu au moins un enfant. Mais cette hâte va très vite s'infléchir. Plus souvent que les autres, cette population vit des unions amoureuses peu confortées par les engagements sociaux ou matériels que sont le mariage et le pacs ou encore le partage d'une vie au quotidien sous un même toit. L'instabilité des liens est telle qu'à un âge moyen alors que les autres femmes rentrent dans un processus de consolidation des relations affectives et familiales, les femmes atteintes par le VIH cumulent les ruptures. L'âge venant, rencontres et séparations continuent à s'enchaîner mais sur un rythme plus lent; la fragilité des liens établis, couplée à la raréfaction des nouvelles rencontres les exposent à un isolement prématuré.
Figure 2

Proportion de femmes n'ayant aucun partenaire dans l'année précédant l'enquête

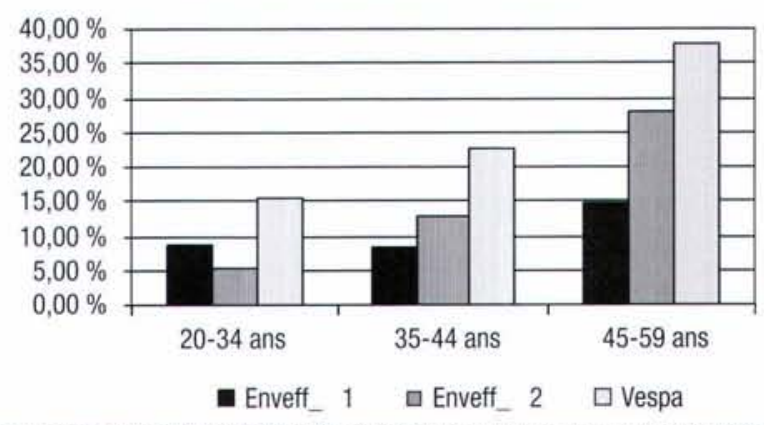

Champ : femmes de nationalité française déclarant une relation au moment de l'enquête, âgées de 20 à 59 ans et ayant répondu à la question.

\section{Une vie sexuelle plus irrégulière et diversifiée}

Très tôt et tout au long de leur vie, les femmes atteintes par le VIH se caractérisent par une vie sexuelle diversifiée, appréciée à partir du nombre et du genre des partenaires sexuels : $63 \%$ d'entre elles déclarent plus de dix partenaires au cours de leur vie, contre $21 \%$ et $18 \%$ dans l'échantillon représentatif de la population féminine française. On retrouve, ici encore, le caractère accéléré des calendriers. Dans la tranche d'âge de 20-34 ans, $55 \%$ des femmes séropositives déclarent plus de cinq partenaires sur la vie contre respectivement $32 \%$ et $19 \%$ dans l'Enveff. Par ailleurs, bien plus souvent que les autres, ces femmes citent au moins une expérience homosexuelle (13\% contre $1 \%$ pour les deux groupes de femmes de l'enquête Enveff).

Cependant, alors qu'en population générale, l'écrasante majorité des femmes qui ont une expérience sexuelle se déclarent sexuellement actives au cours de l'année avec un partenaire exclusif, les femmes atteintes par le VIH ont tendance à se situer aux deux extrêmes : à tout âge, elles sont plus nombreuses à être abstinentes ou, au contraire, multipartenaires. II apparaît qu'avec l'âge, la diminution de l'activité sexuelle soit amplifiée par toute forme d'atteinte sévère de santé ; seule diffère l'ampleur de la mise à l'écart. Si le taux d'inactivité sexuelle des femmes déclarant une pathologie grave dans l'enquête Enveff augmente régulièrement avec l'âge et plus fortement que parmi les femmes " ordinaires ", cet accroissement reste toujours bien en deçà de celui des femmes séropositives avec, pour les plus âgées d'entre elles, une exclusion qui s'avère drastique (figure 2).

Ce rapprochement entre les deux groupes déclarant une atteinte sévère de santé ne joue plus lorsqu'il s'agit du multipartenariat dans l'année. Sur ce point, le comportement des femmes séropositives se distingue fortement de celui des femmes de l'échantillon en population générale : elles sont toujours bien plus nombreuses à déclarer plusieurs partenaires sexuels au cours de l'année qui précède l'enquête (figure 3). 
Figure 3

Proportion de femmes ayant eu plusieurs partenaires dans l'année précédant l'enquête

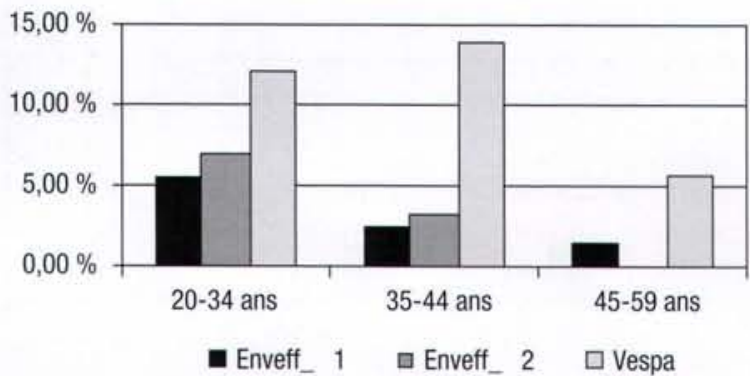

Champ : femmes de nationalité française déclarant une relation au moment de l'enquête, âgées de 20 à 59 ans et ayant répondu à la question.

Deux autres questions attestent de l'irrégularité de leur vie sexuelle. Parmi l'ensemble des femmes de l'échantillon Vespa qui se sont déclarées sexuellement actives au cours de l'année, $26 \%$ disent ne pas avoir eu de rapports sexuels au cours des quatre dernières semaines contre respectivement $14 \%$ et $13 \%$ dans l'Enveff. L'écart s'amplifie avec l'âge : dans la tranche d'âge supérieure, $36 \%$ contre respectivement $15 \%$ et $14 \%$. A cela s'ajoute, pour les femmes atteintes par le VIH ayant une activité sexuelle récente, des déclarations de rapports sexuels plus rares ou, au contraire, plus fréquents au regard de la fréquence modale constatée parmi les femmes de l'Enveff, sans que, sur ce point, n'apparaisse de différenciation majeure liée à l'état de santé.

Par ailleurs, il apparaît que, même au sein d'une relation stable, l'exercice de la sexualité des femmes séropositives reste problématique. Contrairement aux femmes de l'Enveff très majoritairement sexuellement actives avec leur seul conjoint, les femmes atteintes par le VIH engagées dans une relation stable sont plus nombreuses à déclarer des relations extra-conjugales $(11 \%$ contre respectivement $1 \%$ et $2 \%$ ) ou à n'avoir pas eu d'activité sexuelle au cours de l'année ( $8 \%$ contre respectivement $5 \%$ et $2 \%$; et parmi celles qui se sont déclarées sexuellement actives au cours de l'année, $17 \%$ disent ne pas avoir eu de rapports sexuels au cours des quatre dernières semaines contre respectivement $9 \%$ et $7 \%$. A cela s'ajoute des refus plus fréquents de rapports sexuels au sein du couple, que ce soit de leur part ou de celle de leur conjoint. Alors qu'une femme séropositive sur cinq se refuse " souvent » à son conjoint, ce taux est inférieur à une sur vingt parmi les femmes de l'Enveff. Bien que plus rare, la réciproque est également vraie. La dérobade du conjoint face au désir de sa compagne est également plus souvent attestée lorsque celle-ci est séropositive (autour de $5 \%$ contre moins de $1 \%$ pour les deux autres groupes).

En population générale, le poids de l'atteinte grave n'intervient que peu sur la sexualité. Au fil des âges, l'évolution des taux d'activité sexuelle est relativement similaire, à ceci près que la pathologie sévère accélère et amplifie le processus de mise à l'écart. En revanche, les conduites sexuelles des femmes atteintes par le VIH se caractérisent tout à la fois par l'abstinence et la diversité et la multiplicité des rapports sexuels, états contradictoires que l'on ne peut assembler que si l'on raisonne en termes de succession d'épisodes amoureux relativement brefs. Par ailleurs, cette comparaison des caractéristiques de la vie sexuelle des femmes séropositives avec celles de deux groupes de femmes représentatives de la population féminine française constitués sur la base de la déclaration ou non d'atteintes pathologiques graves, n'écarte ni l'idée d'une vie affective et sexuelle qui, en multipliant les rencontres, a pu exposer la femme au risque de contamination par le VIH, ni celle d'une sexualité actuelle non seulement plus diversifiée et moins conventionnelle mais également plus aléatoire. En effet, dans ce domaine, les réactions des femmes séropositives ne sont pas univoques. En se situant au deux extrêmes, y compris au sein de la relation élective, les comportements sexuels de ces femmes rendent compte de leur difficulté à accorder un passé riche en expériences - et qui continue à peser sur le présent - avec une interaction sexuelle perturbée par leur peur de transmettre une infection sexuellement transmissible grave [10].

\section{Seules ou en couple : les parcours de vie}

Les principales caractéristiques des modes de vie des femmes atteintes par le VIH étant dégagées, nous allons maintenant examiner leurs trajectoires affectives et conjugales à partir de leur situation au moment de l'enquête - seule ou en couple - et de la succession de ces épisodes de vie.

\section{Seules}

La comparaison confirme que très tôt ${ }^{4}$, les femmes seules de l'enquête Vespa multiplient les partenaires sexuels et les relations, et que les écarts avec les femmes de l'échantillon en population générale se maintiennent tout au long de leur vie. À ce nombre toujours plus important de relations ayant compté et de partenaires sexuels correspond, à tout âge, un laps de temps de vie en solitaire depuis la dernière rupture toujours plus bref (figure 4).

Si l'on admet que la conjonction de ces trois indicateurs peut être considérée comme un marqueur d'une certaine aptitude à " refaire sa vie ", alors on peut soutenir l'idée que les femmes séropositives font preuve d'une plus grande capacité à rebondir. Cependant la mobilité des femmes atteintes par le VIH ne doit pas faire oublier qu'en dépit d'engagements affectifs et familiaux précoces et souvent renouvelés, dans la tranche d'âge supé-

\footnotetext{
4 Dans la tranche d'âge 20-34 ans, la totalité des jeunes femmes seules séropositives est sexuellement expérimentée (contre respectivement $87 \%$ et $70 \%$ : une majorité cite plus de cinq partenaires sexuels ( $53 \%$ contre respectivement $50 \%$ et $21 \%$ ), la quasi-totalité a fait l'expérience d'une relation affective de plus de six mois (97\% contre respectivement $60 \%$ et $30 \%$ ) et une forte minorité celle de ruptures ( $34 \%$ déclarent plus de trois relations contre respectivement $6 \%$ et $8 \%$ et $19 \%$ ont déjà un statut de e divorcée, séparée ou veuve * contre respectivement $13 \%$ et $3 \%$ en population générale).
} 
Figure 4

\section{Proportion de femmes dont la dernière rupture date de moins d'un an}

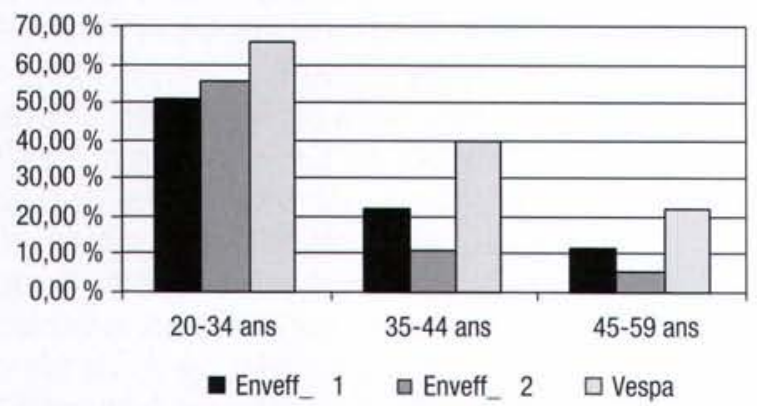

Champ : femmes de nationalité française seules au moment de l'enquête, sexuellement expérimentées, âgées de 20 à 59 ans et ayant répondu à la question.

rieure, le mode de vie des femmes seules atteintes par le VIH est marqué par l'isolement ${ }^{5}$.

En population générale, l'étude des biographies des femmes seules au moment de l'enquête met en exergue deux groupes: celui des jeunes vivant encore avec leurs parents et qui n'ont jamais eu de relation affective de plus de six mois et celui des femmes qui se retrouvent seules après une ou plusieurs ruptures. II apparaît que la solitude de ces dernières est plus conjugale et sexuelle que familiale. Elles déclarent sur leur vie très peu de partenaires sexuels et une, voire au maximum deux relations affectives qui ont compté, et nombre d'entre elles n'ont pas eu de partenaires sexuels au cours de l'année qui précède l'enquête. Avec l'âge, l'absence de relation affective de ces femmes " réservées " s'ancre dans la durée. Pour autant, elles ne sont pas socialement isolées, la rupture les a laissées avec des enfants dont elles s'occupent et elles sont professionnellement actives. Y compris vis-à-vis des produits psycho-actifs, ces femmes sont dans la tempérance, elles consomment parfois de l'alcool mais ne fument ni tabac, ni cannabis. Ici encore, les trajectoires des femmes seules au moment de l'enquête déclarant des atteintes sévères de santé ne se distinguent guère de celles des femmes " témoins ", si ce n'est qu'au déclassement de l'âge s'ajoute celui de l'atteinte. À la suite d'une rupture ou d'un deuil, leur isolement affectif et sexuel s'inscrit dans la durée avec des dates de rupture plus anciennes.

Sur la base d'une succession d'épisodes de vie plus brefs, un autre type d'isolement caractérise les femmes séropositives seules, celui des femmes qui ont multiplié les relations affectives, les expériences et les partenaires sexuels. Elles se distinguent également par l'excès et la diversité de leurs consommations de cannabis, tabac et alcool qu'elles souhaiteraient réduire. Sans surprise, les femmes de ce groupe se caractérisent par une plus

\footnotetext{
$30 \%$ ne se sont jamais mariées contre respectivement $8 \%$ et $8 \%, 29 \%$ n'ont pas d'enfants contre respectivement $9 \%$ et $8 \%, 44 \%$ n'ont pas de relation privilégiée au moment de l'enquête contre respectivement $28 \%$ et $18 \%$, et $42 \%$ vivent seules dans leur logement contre respectivement $15 \%$ et $9 \%$ pour les autres femmes.
}

grande fragilité sociale et psychologique : plus souvent inactives et vivant de minimas sociaux, elles déclarent plus souvent des tentatives de suicide. Pour autant, cette vulnérabilité ne les laisse pas désenchantées, l'expérience est toujours renouvelable comme l'indique la date de leur dernière rupture relativement récente et, plus souvent que les autres, elles ont eu plusieurs partenaires sexuels au cours de l'année qui précède l'enquête. Seuls l'âge et les atteintes physiques auront raison de leur dynamisme.

\section{En couple}

Si l'on s'en tient aux seules femmes qui déclarent une relation affective au moment de l'enquête, la biographie affective et conjugale des femmes atteintes par le VIH s'avère également particulière.

L'état de femme mariée qui devient majoritaire à partir de 35 ans pour les femmes des deux groupes de l'échantillon en population générale, reste une situation minoritaire parmi les séropositives. Quelle que soit la tranche d'âge considérée, la proportion des femmes de l'enquête Vespa mariées ou pacsées est faible et relativement stable ${ }^{6}$. A l'inverse, la situation de concubinage est toujours majoritaire parmi les femmes séropositives engagées dans une relation affective au moment de l'enquête et augmente même avec l'âge, alors qu'en population générale ce mode de vie diminue ${ }^{7}$. II en est de même pour la relation non cohabitante qui se raréfie à partir de 35 ans parmi les femmes de l'Enveff, alors qu'elle augmente avec l'âge parmi les femmes atteintes par le VIH ${ }^{8}$. Corroborant la moindre pérennité des liens établis, il apparaît que très tôt, les femmes séropositives engagées dans une relation au moment de l'enquête ont multiplié les rencontres affectives et les partenaires sexuels, ont divorcé, se sont séparées, ont perdu leur conjoint et qu'au fil des années, les écarts s'accentuent ${ }^{9}$.

La labilité du lien affectif se trouve confirmée par la moindre durée de la relation en cours. Ce constat, valable pour toutes les tranches d'âge, s'accentue, une fois de plus avec l'âge (figure 5). Entre 45 et 59 ans, $76 \%$ des femmes de l'échantillon Enveff sont engagées dans une relation qui dure depuis plus de 20 ans contre seulement $16 \%$ des femmes séropositives au même âge.

\footnotetext{
Passant parmi les sẻropositives de $28 \%$ pour les plus jeunes à $41 \%$ pour les femmes d'âge moyen et $40 \%$ pour les plus âgées, contre respectivement $34 \%, 78 \%$ et $90 \%$ pour les femmes de l'enquête Enveff déclarant un atteinte grave et $48 \%, 81 \%$ et $88 \%$ pour les autres.

7 Dans la tranche d'âge 45-59 ans, $67 \%$ des femmes séropositives déclarant une relation au moment de l'enquête vivent avec un compagnon, contre respectivement $11 \%$ et $12 \%$ en population générale.

8 Dans la tranche d’âge 45-59 ans, $35 \%$ d'entre elles déclarent une relation non cohabitante, contre respectivement $5 \%$ et $6 \%$ dans l'échantillon en population générale.

Entre 45 et 59 ans, $45 \%$ des femmes séropositives en couple ont fait l'expérience d'une rupture ou d'un deuil contre respectivement $7 \%$ et $8 \%, 22 \%$ d'entre elles déclarent au moins trois relations de plus de six mois contre respectivement $4 \%$ et $2 \%$, et $33 \%$ plus de cinq partenaires sexuels contre respectivement $3 \%$ et $3 \%$ dans l'échantillon représentatif de la population féminine française.
} 
Figure 5

Pourcentage de femmes dont la durée de couple est supérieure à 10 ans

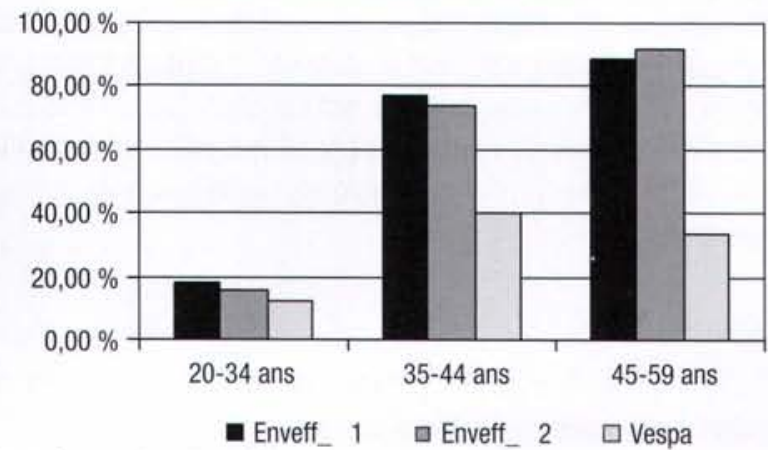

Champ : femmes de nationalité française, en couple au moment de l'enquête, âgées de 20 à 59 ans et ayant répondu à la question.

L'étude des biographies selon l'âge des femmes en couple au moment de l'enquête met en évidence deux parcours :

- un parcours temporel pour les femmes des deux groupes de l'échantillon en population générale, qui va des plus jeunes déclarant une relation stable non cohabitante et qui se transforme rapidement en une cohabitation avec un compagnon. À partir de 30 ans, le mode de vie se caractérise par l'insertion professionnelle et la vie de famille (activité professionnelle, vie commune avec son mari et deux enfants ou plus au foyer). L'activité sexuelle est régulière et sans réticence marquée de la part de l'un ou l'autre des conjoints. Ce processus d'installation dans la conjugalité est concordant avec une augmentation de la durée du couple selon l'âge au moment de l'enquête ;

- un parcours indépendant de l'âge pour les femmes séropositives, qui se caractérise par une multiplication des rencontres et des expériences sexuelles, une fragilité de la vie conjugale (concubinage, non cohabitant), de la vie familiale (vie en solo, séparée ou divorcée, foyer monoparental) et des conditions économiques (minima sociaux). La contingence et la fragilité de leurs relations et de leur situation économique sont associées à des tentatives de suicide plus fréquentes, une consommation de cannabis et d'alcool qu'elles estiment nécessaire de réduire.

Les femmes en couple de l'Enveff déclarant des atteintes graves de santé ne se distinguent guère des femmes " témoins " sauf par leur âge. Contrairement au profil de vie des femmes séropositives, elles ont une trajectoire affective qui s'est installée dans la durée.

\section{Des parcours de vie plus ou moins différenciés}

Au-delà des différences comportementales, comment interpréter les variations de temporalités observées? L'analyse en termes d'épisodes de solitude et de conjugalité statistiquement plus ou moins courts ou plus ou moins longs nous conduit à poser autrement la comparaison des parcours de vie entre les femmes séropositives et les autres.

Pour les femmes de l'Enveff, en raison d'épisodes de vie bien plus longs, le fait d'être en couple ou seule au moment de l'enquête présume de parcours affectifs et sexuels spécifiques. Celles qui sont en couple sont nombreuses à déclarer un seul partenaire sexuel sur la vie, leur conjoint. Ce type de trajectoire s'oppose à celui des femmes qui sont seules avec une longue vie en solo derrière elles. Sur la base de périodes de vie en couple ou seule relativement longues et d'alternances plus rares entre les deux états, la situation affective des femmes de l'échantillon aléatoire au moment de l'enquête induit des trajectoires de vie très différenciées. Cette différenciation n'est pas aussi nette parmi les femmes séropositives. Dans ce groupe, la brièveté des temps dans chacune des situations et la répétition des expériences précipitent l'alternance entre épisodes de solitude et de conjugalité, diminuant ainsi la probabilité de carrières affectives et sexuelles nettement séparées.

\section{Conclusion}

Pour les femmes en population générale, l'existence ou non d'une relation stable au moment des enquêtes renvoie à des situations qui s'inscrivent dans la durée. Cela est moins vrai pour les femmes séropositives : dans ce groupe, statuts incertains et provisoires, fortes probabilités de changement et frontières qui se brouillent, contribuent à affaiblir la dissemblance entre parcours de vies en solo et en couple constatée en population générale.

Seules ou en couple, les femmes de l'Enveff sans atteinte grave de santé sont dans un processus de conservation affective. Selon les codes sociaux en usage, pour les femmes en couple, stabilité et sécurité d'ordre domestique sont atteintes tandis que pour les femmes désormais seules, l'ordre affectif et conjugal s'est défait sans que soient intégrées la conscience et la reconnaissance de la fragilité des relations humaines ; bien que désormais " libres ", elles restent dans une logique de tempérance.

Les parcours biographiques des femmes atteintes de maladies ou de handicaps graves ou chroniques de l'échantillon en population générale sont à la fois ordinaires et particuliers ; globalement leur histoire est celle de la majorité, elles sont dans des logiques de conservation affective; elles en diffèrent cependant quant au rythme, avec, pour les plus jeunes, un calendrier en accéléré auquel succède, pour les plus âgées, un déclassement lui aussi plus rapide. Elles en diffèrent également par des épisodes de vie plus pérennes. Après la construction d'une vie conjugale qui s'inscrit au départ dans la durée, une fois ce lien délié, ces femmes, plus souvent et plus longtemps que les autres, restent seules sans refaire leur vie.

Au contraire, les femmes séropositives, sur fond de situation économique difficile et d'une vie qui se fait et se défait constamment, cumulent les handicaps de leur groupe d'origine et de leur genre, 
auxquels s'ajoutent ceux de la maladie. Leurs parcours affectifs s'inscrivent dans un monde flottant, une logique d'errance, entre désaffection et engagement inconditionnel qui renvoie à la "notion de dépense " selon Bataille [11]. Ces femmes ne parviennent à se couler de manière un peu stable et durable ni dans la vie professionnelle ni dans la vie affective. Elles se caractérisent par l'instabilité et la précarité de leurs engagements, sans avoir pu tirer les bénéfices de leurs expériences. Au fur et à mesure que les femmes avancent en âge et que les ruptures et les rencontres se répètent, leurs possibilités d'intégration s'altèrent : le temps de l'installation n'est plus et les opportunités de rencontre, avec la possibilité de fonder des relations stables, se raréfient.

Le présent des femmes séropositives, tributaires au même titre que les autres femmes de la reconnaissance d'autrui, apparaît comme plus lourdement obéré par leur passé. Outre le déclassement ordinaire lié à l'âge, leur style de vie actuel est l'expression de la tension entre la rémanence de leur appétence de vie passée et un présent tourmenté par la conscience de leur responsabilité vis-à-vis d'une possible contamination de leur partenaire sexuel [10]. À toutes ces difficultés, s'ajoute le déclassement d'une infection sexuellement transmissible qui dévalorise leur corps et jette un soupçon sur leur conduite. Ces femmes cumulent les états et les conduites en marge des situations et des comportements conventionnels et valorisés, contrevenant au mode de vie couramment admis comme normal et naturel [12].

Certes, notre approche masque la diversité des parcours et des adaptations des personnes atteintes par le $\mathrm{VIH}$ mise en valeur par J Pierret [13]. Mais en ne mettant en exergue que les traits statistiques les plus saillants du groupe nous pensons pouvoir rendre compte de ce que la perception collective peut également appréhender. L'acceptabilité des conduites et des modes de vie de ces femmes ainsi perçue est alors évaluée à l'aune des valeurs traditionnelles qui régissent la "bonne" conduite des femmes. Comme on l'a vu, les femmes séropositives présentent des trajectoires de vie affective et sexuelle singulières par rapport à celles déclarant des atteintes graves. Sans changement de mentalités, les stigmates d'un corps perçu comme dangereux et ceux d'un corps suspect, en référence à des mode de vie réprouvés et des conduites instables, grèvent lourdement la possibilité pour ces femmes d'être considérées comme des malades comme les autres [14].

\section{Références bibliographiques}

1. Pierret J. Un objet pour la sociologie de la maladie chronique : la situation de séropositivité au VIH. Sciences Sociales et Santé 1997 ; 15 (4) : 98-120.

2. Setbon M. La normalisation paradoxale du sida. Revue Française de Sociologie $2000 ; 41-1: 61-78$.

3. Martin C. Trajectoires post-divorce et vulnérabilité. In L'exclusion : l'état des savoirs, S Paugham, Ed. Paris : Editions La Découverte, 1996 : 172-182.

4. Lert F, Obadia Y et l'équipe de l'enquête Anrs-Vespa. Comment vit-on en France avec le $\mathrm{VIH} /$ sida? Population et Société $2004 ; 406: 4 \mathrm{p}$.

5. Jaspard M, Brown El, Condon S, Fougeyrollas-Schwebel D, Houel A, Lhomond B, Maillochon F, Saurel-Cubizolles MJ, Schiltz MA. Les violences envers les femmes en France. Une enquête nationale. Paris : La Documentation française, 2003.

6. Dray-Spira R, Gueguen A, Ravaud JF, Lert F. Socioeconomic differences in the impact of HIV infection on workforce participation in France in the era of highly active antiretroviral therapy. American Journal of Public Health 2007 ; 97 :552558.

7. Castel R. Dossier "la société précaire ». Sciences humaines, février 2006, 168.

8. Drees (Direction de la recherche, des études, de l'évaluation et des statistiques). Les familles monoparentales et leurs conditions de vie. Études et Résultats $2005 ; 389: 12 \mathrm{p}$.

9. Giami A. L'expérience de la sexualité. In L'expérience de la sexualité chez les jeunes adultes. Entre errance et conjugalité, A Giami, MA Schiltz, Eds. Paris: Inserm, collection Questions de santé publique, 2004, p. 1-21.

10. Schiltz MA, Bouhnik AD, Préau M, Spire B et le groupe d'étude Anrs-EN12Vespa. La sexualité des personnes atteintes par le VIH: l'impact d'une infection sexuellement transmissible. Sexologie 2006 ; 15 : 157-164.

11. Bataille G. La part maudite précédé de La notion de dépense. Paris: Les Editions de Minuit, 1967 (Critique).

12. Schiltz MA. L'intolérable indépendance de la femme publique. In Violences envers les femmes: trois pas en avant, deux pas en arrière, A Giami, MA Schiltz, Eds. Paris : L'Harmattan, collection Bibliothèque du féminisme, $2007,320 \mathrm{p}$.

13. Pierret J. Vivre avec le VIH : enquête de longue durée auprès des personnes infectées par le VIH. Paris: Presses universitaires de France, collection Le lien social 2006.

14. Théry I. « Une femme comme les autres ", séropositivité, sexualité et féminité. In Séropositivité, vie sexuelle et risque de transmission du VIH, F Lert, Y Souteyrand, Eds. Paris : Anrs, collection Sciences sociales et sida, 1999, 113-136. 